\title{
Tissue distribution of SARS-CoV-2 in non-human primates after combined intratracheal and intranasal inoculation
}

\author{
Tian-Zhang Song ${ }^{1 \dagger}$, Jian-Bao $\operatorname{Han}^{2 \dagger}$, Xiang Yang ${ }^{2 \dagger}$, Ming-Hua $\mathrm{Li}^{2}$ \& Yong-Tang Zheng ${ }^{1,2}$ \\ ${ }^{1}$ Key Laboratory of Animal Models and Human Disease Mechanisms of the Chinese Academy of Sciences/Key Laboratory of Bioactive Peptides \\ of Yunnan Province, KIZ-CUHK Joint Laboratory of Bioresources and Molecular Research in Common Diseases, Kunming Institute of \\ Zoology, Chinese Academy of Sciences, Kunming 650223, China; \\ ${ }^{2}$ Kunming National High-level Biosafety Research Center for Non-human Primates, Center for Biosafety Mega-Science, Kunming Institute of \\ Zoology, Chinese Academic of Sciences, Kunming 650107, China
}

Received November 10, 2020; accepted December 14, 2020; published online February 10, 2021

Citation: Song, T.Z., Han, J.B., Yang, X., Li, M.H., and Zheng, Y.T. (2021). Tissue distribution of SARS-CoV-2 in non-human primates after combined intratracheal and intranasal inoculation. Sci China Life Sci 64, 1792-1794. https://doi.org/10.1007/s11427-020-1877-4

\section{Dear Editor,}

As of 5 July, 2020, approximately 20,162,000 confirmed cases of coronavirus disease 2019 (COVID-19), which is caused by severe acute respiratory syndrome coronavirus 2 (SARS-CoV-2), have been reported globally, including an estimated 740,000 deaths. Although the clinical manifestations of COVID-19 are heterogeneous, viral infection and pathological changes in the respiratory system, especially the lungs, still represent common characteristics of COVID-19. The nose, served as the entrance to the respiratory system, seems to produce absent or minimal symptoms of nasal congestion or rhinorrhea during SRAS-CoV-2 infection, while other viral rhinitis (i.e., adenovirus, rhinovirus, influenza virus, etc.) are characterized by typical nasal symptoms, including nasal congestion, rhinorrhea, and sneezing. Recent studies suggest that, despite a lack of other nasal symptoms, anosmia is an important sign of early-stage COVID-19 (Lechien et al., 2020). In addition, Sungnak et al. (2020) reported that SARS-CoV-2 receptors are highly expressed in nasal epithelial cells together with innate immune genes, suggesting that these cells can be infected by SARS-CoV-2 and may play a potential role in transmitting infection to the

$\dagger$ Contributed equally to this work

*Corresponding author (email: zhengyt@mail.kiz.ac.cn) rest of the body. Furthermore, based on infection data from young children with ACE2 lower expression in the nasal epithelium, Patel and Verma (2020) found that while COVID-19 morbidity is similar between middle-aged adults and younger children, young children with SARS-CoV-2 infection show mild clinical manifestations compared with middle-aged adults. Thus, these results suggest that the importance of nasal infection has been primarily ignored in earlier studies. Moreover, nasal infection may influence SARS-CoV-2 transmission or infectious status in the lungs.

We previously established a successful SARS-CoV-2-infected non-human primate model (Shan et al., 2020; Song et al., 2020). To evaluate the characteristics of nasal infection in COVID-19, six healthy Chinese rhesus macaques (male, adult, $3-7 \mathrm{~kg},<18$ years old) were enrolled in this study. Three macaques (No. 15011, 02059, and 04305) were inoculated intratracheally with a $1 \times 10^{7}$ mean tissue culture infective dose $\left(\mathrm{TCID}_{50}\right)$ SARS-CoV-2 and three macaques (No. 14377, 17007, and 15007) were inoculated with $1 \times 10^{7}$ $\mathrm{TCID}_{50}$ SARS-CoV-2 intratracheally $\left(60 \%, 6 \times 10^{6} \mathrm{TCID}_{50}\right)$ and intranasally $\left(40 \%, 4 \times 10^{6} \mathrm{TCID}_{50}\right)$ (Figure 1A). The intratracheal inoculation was achieved using a bronchoscope. Subsequently, $200 \mu \mathrm{L}$ of viral suspension $\left(2 \times 10^{6} \mathrm{TCID}_{50}\right)$ was respectively injected into the two nostrils using a $1 \mathrm{~mL}$ needle-free syringe. All animals were euthanized for tissue collection on the 7 th day post infection (7 dpi). As shown in 

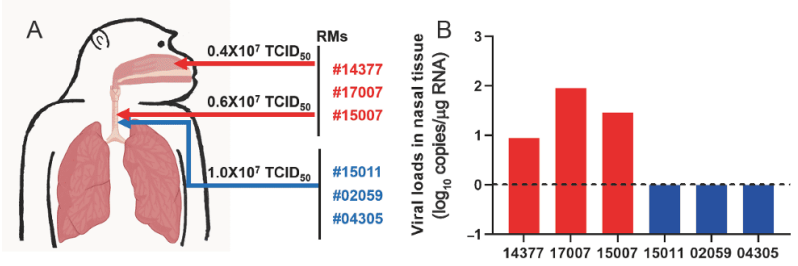

C Nose Swabs

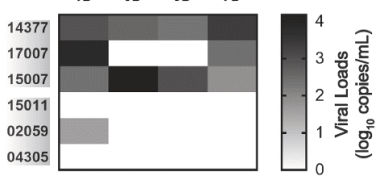

D

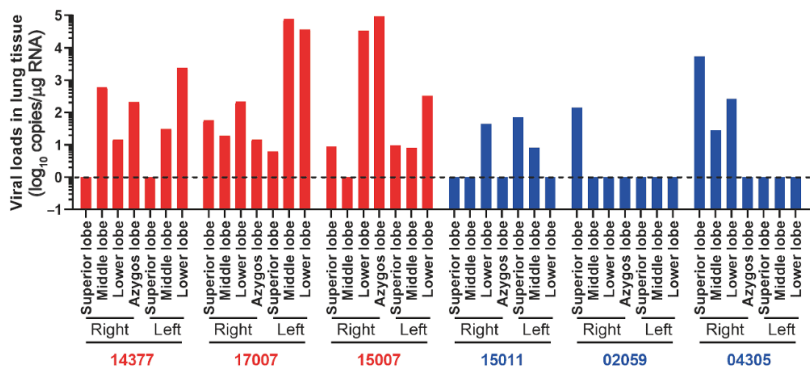

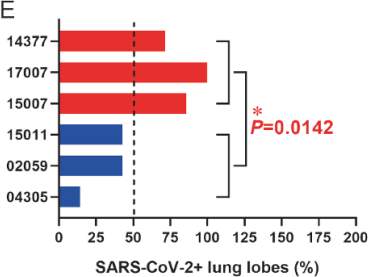
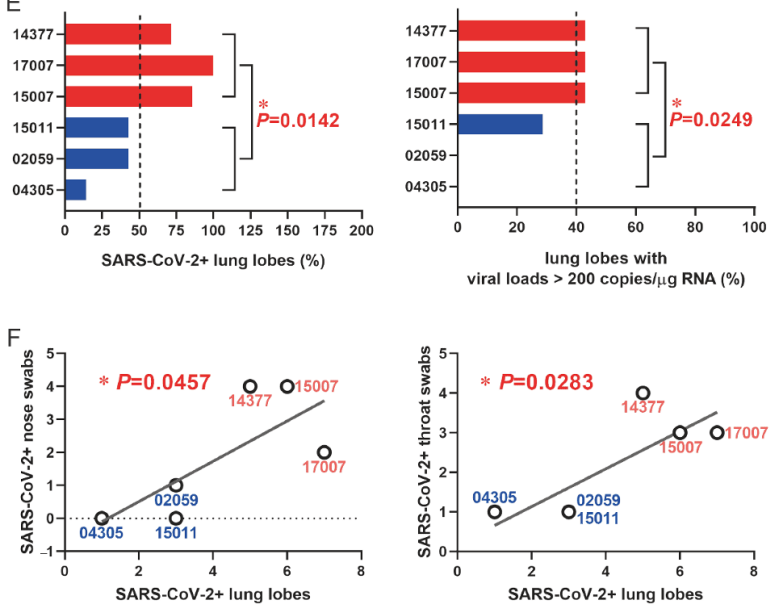

G
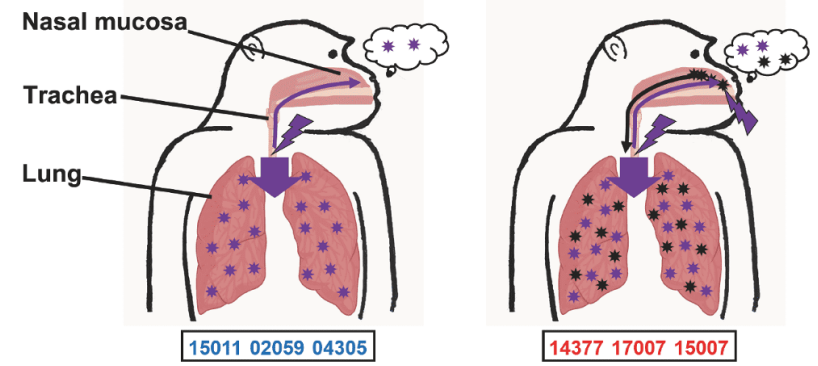

Figure 1 Nasally infectious characteristics in SARS-CoV-2-infected rhesus macaques. A, Inoculation route of Chinese rhesus macaques. B, Viral loads in nasal tissue. C, Viral loads in nose and throat swabs. D, Viral loads in lung lobes. E, Analysis of viral loads in lung lobes. F, Correlation analysis between SARS-CoV-2+ swabs and SARS-CoV-2+ lobes. G, Schematic of differences between single and two-way inoculation. Unpaired $t$-test was used to compare viral loads in the lungs between nose infection and non-infection groups. Pearson's rank test was used to determine correlations between positive lobes and positive swabs. ${ }^{*} P<0.05$ was considered statistically significant. All data analyses were performed using GraphPad Prism v8 (GraphPad Software, USA).

Figure 2B, viral RNA was detected in the nasal tissues of all macaques receiving intratracheal and intranasal inoculation (i.e., No. 14377, 17007, and 15007), whereas those macaques receiving only intratracheal inoculation (i.e., No. 15011, 02059, and 04305) showed negative viral RNA results in the nasal tissues. Subsequently, we divided the macaques into a nasal infection group (marked red) and nasal non-infection group (marked blue). Nose and throat swabs were collected on $1,3,5$, and $7 \mathrm{dpi}$. The positive ratios of viral RNA in the nasal infection group were much higher than that in the nasal non-infection group in both swab types (Figure 1C), suggesting more virus particles congregated in the upper respiratory tract of macaques with nasal infection. Subsequently, viral loads in seven lobes of the lung were detected to evaluate infectious status in the lower respiratory tract (Figure 1D). The ratios of lobes with positive viral RNA were markedly higher in the nose infection group than in the nose non-infection group (Figure 1E). In addition, the percentages of lobes with viral loads $>200$ copies $\mu \mathrm{g}^{-1}$ RNA were significantly higher in the nose infection group than in the nose non-infection group (Figure 1E). These differences in the lungs indicated that nasal infection aggravated viral transmission and replication in the lower respiratory tract, suggesting more severe viral infection in the nasal infection group. As the respiratory tract exhibits obvious continuity and consistency in structure and function, the infectious status relationship between the upper and lower respiratory tract was explored by analyzing the correlation between the ratios of SARS-CoV-2+ swabs and percentages of SARSCoV-2+ lung lobes. As seen in Figure 1F, infectious status showed positive correlation between the upper and lower respiratory tracts. Therefore, nasal SARS-CoV-2 infection markedly enhanced the number of virus particles in the whole respiratory airway, leading to more severe viral infection in the lungs and wider viral transmission.

Zheng et al. (2020) previously established a SARS-CoV-2infected rhesus macaque model via single nasal inoculation. Viral loads could be detected in the kidney, liver, spleen, heart, intestine, testis, brain, spinal cord, and lymph nodes, suggesting that nasal infection with SARS-CoV-2 can induce wide viral dissemination in rhesus macaques. However, the viral loads in the lung tissues were relatively low, ranging from $10^{2}$ to $10^{3}$ copies $/ 100 \mathrm{mg}$ tissue, with peak loads much lower than that in the lymph nodes, trachea, liver, and spleen. In addition, the presence of viral RNA in lung tissue displayed obvious variations among animals. Therefore, SARS- 
CoV-2 infection via single nasal inoculation showed wide viral dissemination but atypical infection in the lungs, which differs from the clinical manifestations observed in patients with COVID-19. Our results indicate the importance of nasal infection in COVID-19, as summarized in Figure 1G. Single intratracheal inoculation induced heterogeneous lung infection and minimal transmission to the upper respiratory tract. However, nasal and intratracheal infection enhanced the number of virus particles of both the upper and lower respiratory tracts. Thus, the severity of respiratory tract infection enhanced air transmission and induced wide and severe SARS-CoV-2 infection in the lungs, suggesting the occurrence of more severe infectiousness and lung injury in SARS-CoV-2 patients with nasal infection. Therefore, nasal infection may be regarded as an important index to assess the severity of COVID-19. Furthermore, treatments focused on nasal infection, like hypertonic saline nasal irrigation and gargling (Ramalingam et al., 2020), should be considered as a therapeutic option for COVID-19.

Compliance and ethics The author(s) declare that they have no conflict of interest. All animal experiments were performed in the Kunming $\mathrm{Na}$ tional High-Level Biosafety Research Center for Non-Human Primates, Center for Biosafety Mega-Science, KIZ, CAS, according to the guidelines of the Committee on Animals of KIZ, CAS (approval No.: IACUC20005). All authors state that they conformed with the Helsinki Declaration of 1975 concerning Human and Animal Rights.
Acknowledgements This work was supported by the National Key Research and Development Program of China (2020YFC0842000).

\section{References}

Lechien, J.R., Chiesa-Estomba, C.M., De Siati, D.R., Horoi, M., Le Bon, S. D., Rodriguez, A., Dequanter, D., Blecic, S., El Afia, F., Distinguin, L., et al. (2020). Olfactory and gustatory dysfunctions as a clinical presentation of mild-to-moderate forms of the coronavirus disease (COVID-19): a multicenter European study. Eur Arch Otorhinolaryngol 277, 2251-2261.

Patel, A.B., and Verma, A. (2020). Nasal ACE2 levels and COVID-19 in children. JAMA 323, 2386.

Ramalingam, S., Graham, C., Dove, J., Morrice, L., and Sheikh, A. (2020). Hypertonic saline nasal irrigation and gargling should be considered as a treatment option for COVID-19. J Glob Health 10, 010332.

Shan, C., Yao, Y.F., Yang, X.L., Zhou, Y.W., Gao, G., Peng, Y., Yang, L., Hu, X., Xiong, J., Jiang, R.D., et al. (2020). Infection with novel coronavirus (SARS-CoV-2) causes pneumonia in rhesus macaques. Cell Res 30, 670-677.

Song, T.Z., Zheng, H.Y., Han, J.B., Jin, L., Yang, X., Liu, F.L., Luo, R.H., Tian, R.R., Cai, H.R., Feng, X.L., et al. (2020). Delayed severe cytokine storm and immune cell infiltration in SARS-CoV-2-infected aged Chinese rhesus macaques. Zool Res 41, 503-516.

Sungnak, W., Huang, N., Bécavin, C., Berg, M., Queen, R., Litvinukova, M., Talavera-López, C., Maatz, H., Reichart, D., Sampaziotis, F., et al. (2020). SARS-CoV-2 entry factors are highly expressed in nasal epithelial cells together with innate immune genes. Nat Med 26, 681687.

Zheng, H., Li, H., Guo, L., Liang, Y., Li, J., Wang, X., Hu, Y., Wang, L., Liao, Y., Yang, F., et al. (2020). Virulence and pathogenesis of SARSCoV-2 infection in rhesus macaques: a nonhuman primate model of COVID-19 progression. PLoS Pathog 16, e1008949. 